“(C) 2014 IEEE. Personal use of this material is permitted. Permission from IEEE must be obtained for all other uses, in any current or future media, including reprinting/republishing this material for advertising or promotional purposes, creating new collective works, for resale or redistribution to servers or lists, or reuse of any copyrighted component of this work in other works." 


\title{
A Price Prediction Model for Online Auctions using Fuzzy Reasoning Techniques
}

\author{
Preetinder Kaur, Madhu Goyal, and Jie Lu
}

\begin{abstract}
E-consumers are urged to opt for the best bidding strategies to excel in the competitive environment of multiple and simultaneous online auctions for same or similar items. It becomes very complicated for the bidders to make the decisions of selecting which auction to participate in, place single or multiple bids, early or late bidding and how much to bid. In this paper, we present the design of an autonomous dynamic bidding agent (ADBA) that makes these decisions on behalf of the buyers according to their bidding behaviors. The agent develops a comprehensive methodology for initial price estimation and an integrated model for final price prediction. The initial price estimation methodology selects an auction to participate in and assesses the value (initial price) of the auctioned item. Then the final price prediction model forecasts the bid amount by designing different bidding strategies using fuzzy reasoning techniques. The experimental results demonstrated improved initial price prediction outcomes by proposing a clustering based approach. Also, the results show the proficiency of the fuzzy bidding strategies in terms of their success rate and expected utility.
\end{abstract}

\section{INTRODUCTION}

$\mathrm{T}$ HE advent of electronic commerce has dramatically advanced the traditional trading mechanism i.e. online auctions and has turned up as a powerful tool to allocate goods and resources. In the online auctions' enterprise, traders purchase or sell products by employing specific trading rules over the internet supporting different auction formats. The common online auction formats are English auction; Dutch auction; First-price sealed-bid; and Secondprice sealed-bid [1, 2]. English auctions are the most common auction type employed by the online auctioneers in eBay, Amazon etc. Bidders participating in this marketplace strive for choosing the most favorable bidding strategies to win the auction based on their bidding behavior. Moreover, there are multiple auctions where bidders can obtain their desired items. This further complicates the situation of the bidders in making decisions of selecting which auction to participate in, placing single or multiple bids and how much to bid $[3,4]$. These hard and time consuming processes of analyzing, selecting and making bids and monitoring are needed to be automated to assist the buyers while bidding. Software agents can promisingly act upon these tasks on behalf of the traders. These are the software tools that can execute autonomously, communicates with other agents or the user and monitors the state of its execution environment effectively. These negotiating agents outperform their human counterparts because of their systematic approach to execute complex decision making situations [5, 6]. The software agents make decisions on behalf of the consumers and endeavor to guarantee the delivery of the item according to their bidding behavior.

eBay style auctions adopt the English auction format with an exception to the winning bid [7]. In eBay auctions, a bidder with the highest value wins and he pays the secondhighest bid plus one bid increment amount instead of paying his own bid. However, the bidders do not bid their maximum values either because they have trouble understanding that they should bid their maximum value or they have trouble simply figuring out what their maximum value is. Closing price prediction of the auction can help the bidders in setting their maximum valuation of the auctioned item. Furthermore, the bidders adjust their bids towards the maximum valuation of the item repeatedly in response to the remaining time left of the auction and the bids placed by the other participants, which leads to the different bidding behaviors. According to different bidding behaviors, bidders are categorized as evaluators, participators, opportunists, skeptics, snipers, unmaskers or shill bidders [8-10]. Moreover, late bidding in online auctions has aroused a good deal of attention [11-13]. Late bidders appear near to the closing hours of an auction. This is a very common behavior by the bidders in an eBay style auction with hard closing rules. Late bidding may be the best response to a variety of incremental bidding strategies because they can better realize the state of the auction by observing its historical data. So there is a need to design a mechanism which decides the bid amount at a particular moment in time according to the bidding behavior of the late bidders.

The closing price prediction of an online auction is critical because of the dynamic nature of the auction attributes [14]. It depends on the path followed by the price during the auction which is known as price dynamics of the auction. Different data mining techniques has been used to predict the closing price of an auction. Data from a series of the same or similar auctions closed in the past has been used to forecast the winning bid by exploiting regression, classification and regression tree, multi-class classification and multiple binary classification tasks an auction [15-18]. Also the history of an ongoing auction contains significant information and is exploited for the short term forecasting of the next bid by using support vector machines, functional k-nearest neighbor, clustering, regression and classification techniques $[14,16,19]$. However, most of these price prediction methods are static that uses information which is available only in the beginning of the auction $[18,20]$. These cannot incorporate price dynamics of auctions for similar items like the models in [21-23]. In addition, price dynamics of auctions are different, even when dealing with the auctions 
of similar items which has a strong influence on the price prediction outcomes [24]. So there is a need to characterize these auctions based on their price dynamics before predicting the closing price of the auction.

In this article an Automated Dynamic Bidding Agent ADBA is developed that uses machine learning and fuzzy reasoning techniques for bidding in an environment of multiple and simultaneous online auctions of same or similar items. This agent assists bidders in making decisions by designing bidding strategies as per their different bidding behaviors. ADBA selects an auction to participate in and assesses the value of the item by adopting a clustering based bid mapping and selection approach. The final price of the selected auction is predicted by designing bidding strategies based on bidding behavior of bidders using Mamdani's Method for fuzzy relations and compositional rule of inference [25].

The rest of the paper is organized as follows. In section II we present the design of the Automated Dynamic Bidding Agent. Section III depicts experimental results evaluating the performance of the closing price prediction methodology and the success rate of the bidding strategies designed for different bidding behaviors of the bidders. Section IV concludes the paper.

\section{II.AUTOMATED DYNAMIC BIDDING AGENT-ADBA}

The automated dynamic bidding agent (ADBA) is represented in Fig. 1. The bidding agent predicts the final price of the auction in two phases: phase 1 for the initial price estimation and phase 2 for the final price prediction. Phase 1 is responsible for the selection of an ongoing auction for participation and assessing the value of the item in the selected auction. This assessed value appears as the initial price during the second phase of the bidding agent. Phase 2 utilizes this initial price in order to predict the final price of the auction based on different bidding strategies of the bidders.

\section{A. Phase 1: Initial Price Estimation}

The first phase of the ADBA agent estimates the initial price of an auction and is formally consists of three steps. First, similar auctions are clustered together in $k$ groups based on their price dynamics. Second, an auction for participation is selected by nominating a cluster for each ongoing auction using a bid mapping and selection technique. Third, the value of the item in the selected auction is assessed using machine learning techniques.

1) Cluster Analysis: Let ATT be the set of the attributes collected for each auction then $A T T=\left\{a_{1}, a_{2} \ldots . a_{j}\right\}$ where $j$ is the total number of attributes. Different types of auctions are categorized based on some predefined attributes from the vast feature space of online auctions. The feature space may include average bid amount, average bid rate, number of bids, item type, seller reputation, opening bid, closing bid, quantity available, type of auction, duration of the auction, buyer reputation and many more. In this paper, to classify different types of auctions, we focus on only a set of attributes; opening bid, closing price, number of bids, average bid amount and average bid rate. Now $A T T=\left\{\right.$ OpenB $_{i}$, CloseP $\left._{i}, N U M_{i}, A v g B_{i}, A v g B R_{i}\right\}$,

where $A T T$ be the set of attributes for an auction

Open $B_{i}$ be the opening bid of the $i^{\text {th }}$ auction

Close $P_{i}$ be the closing price of the $i^{\text {th }}$ auction

$N U M_{i}$ be the total number of bids placed in the $i^{\text {th }}$ auction

$A v g B_{i}$ be the average bid amount of the $i^{\text {th }}$ auction and can be calculated as $\operatorname{Avg}\left(B_{1}, B_{2}, \ldots . B_{l}\right)$ where $B_{1}$ is the 1 st bid amount, $B_{2}$ is the second bid amount and $B_{l}$ is the last bid amount for the $i^{\text {th }}$ auction.

$A v g B R_{i}$ be the average bid rate of the $i^{\text {th }}$ auction and can be calculated as

$$
\operatorname{AvgBR}_{i}=\frac{1}{n} \sum_{i=1}^{n} \frac{B_{i+1}-B_{i}}{t_{i+1}-t_{i}}
$$

where $B_{i+1}$ is the amount of $(i+1)^{\text {th }}$ bid, $B_{i}$ the amount of $i^{\text {th }}$ bid, $t_{i+1}$ is the time at which $(i+1)^{\text {th }}$ bid is placed and $t_{i}$ is the time at which $i^{\text {th }}$ bid is placed.

Deciding the value of $k$ in $k$-means algorithm is a recurrent problem in clustering and is a distinct issue from the process of actually solving the clustering problem. The optimal choice of $k$ is often ambiguous, increasing the value of $k$ always reduce the error and increases the computation speed. The most favorable method to find $k$ adopts a strategy which balances between maximum compression of the data using a single cluster, and maximum accuracy by assigning each data point to its own cluster. In this paper, the value of $k$ in $k$ means algorithm is determined by employing elbow method using one way analysis of variance (ANOVA) [26].

Once we decide the value of $k, k$-means clustering algorithm is used to partition the similar auctions based on their characteristics. Given a set $A$ of $N$ auctions $A=\left\{a_{1}, a_{2}, \ldots a_{N}\right\}$ where each auction is 5-dimensional real vector $A T T=\left[\operatorname{OpenB}_{i}, C_{\text {loseP }}, N_{U} M_{i}, A v g B_{i}, A v g B R_{i}\right], k$ means clustering aims to partition $N$ auctions into $k$ clusters $(k<N)$ in such a way which minimize the within-cluster dispersion. The within-cluster dispersion is the sum of squared euclidean distances of auctions from their cluster centroid [26].

2) Bid Mapping and Selection: In order to decide that the current ongoing auctions belong to which cluster, the bid mapping and selection component is activated. Based on the transformed data after clustering and the characteristics of the current auctions, it nominates the cluster for each of the ongoing auction to select the auction for participation.

We observe that in 92 of the 149 auctions of our dataset, winner first appear in the last hour of the auction, which account for the $62 \%$ of the total auctions, consistent with the late bidding attitude of the bidders recognized in the online auction literature [11-13]. Also, clustering has divided the auction data into the groups of auctions having distinct range of average bid rate $(A v g B R)$ values. Furthermore, it has been observed that the value of $A v g B R$ in $78 \%$ of the completed auctions belong to the same cluster as at the beginning of the last hour of the auction. So a Bid Mapper and Selector (BMS) algorithm is proposed for selecting the target auction 
for participation which maps the ongoing auctions to the clusters based on their $A v g B R$ value in the beginning of the last hour.

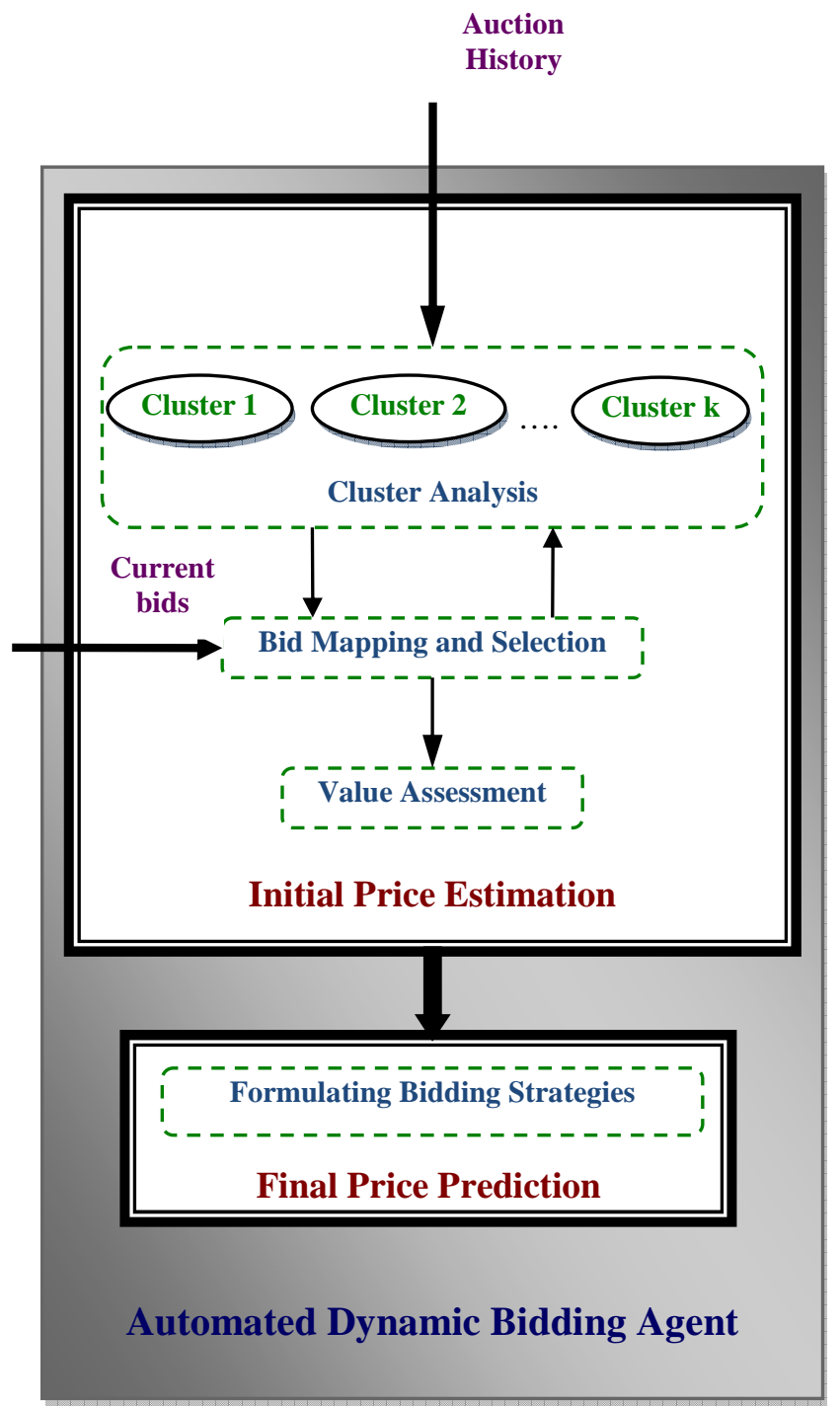

Fig. 1. Automated Dynamic Bidding Agent

Given a set of ongoing auctions $O A=O A_{1} U O A_{2}$ $U \ldots \ldots . U O A_{k}$. where $O A_{i}=\left[O A_{i 1}, O A_{i 2}, \ldots \ldots O A_{\text {in }}\right], O A_{i}$ is the set of ongoing auctions belonging to the $i^{\text {th }}$ cluster , $i=1,2, \ldots . k$ where $k$ is the total number of clusters and $n$ is the total number of ongoing auctions belonging to the $i^{\text {th }}$ cluster, BMS algorithm selects a subset of $O A$ if $A v g B_{k n}<$ $A v g B C_{k}$, where $A v g B_{k n}$ is the average bid amount of the $n^{\text {th }}$ auction in $k^{\text {th }}$ cluster and $A v g B C_{k}$ is the average bid amount of the $k^{\text {th }}$ cluster.

The target auction $T A$ is selected which gives maximum surplus to the bidders. Surplus is the bidders' returns by winning an auction at a price lower than its predicted closing price [23]. The auctions with the lower average bid amounts are expected to give more surplus to the bidders. So the target auction $T A$ is selected for participation with least average bid amount in order to maximize the bidders' surplus. The developed algorithm is presented in Fig. 2.

\author{
$K \leftarrow$ total number of clusters \\ $N \leftarrow$ total number of auctions \\ Cnt $t_{k} \leftarrow$ counts the number of auctions in the $k^{\text {th }}$ cluster \\ AvgBROA $A_{i} \leftarrow$ average bid rate of the $i^{\text {th }}$ ongoing auction \\ MinAvgBRC $C_{k} \leftarrow$ minimum average bid rate of the \\ auctions in the $k^{\text {th }}$ cluster \\ MaxAvgBRC $C_{k} \leftarrow$ maximum average bid rate of the \\ auctions in the $k^{\text {th }}$ cluster \\ $O A_{k} \leftarrow$ set of auctions in the $k^{\text {th }}$ cluster \\ $A v g B C_{k} \leftarrow$ average bid amount of the auctions in the $k^{\text {th }}$ \\ cluster \\ $T A \leftarrow$ target auction for participation \\ $A v g B_{T A} \leftarrow$ average bid amount of the target auction
}

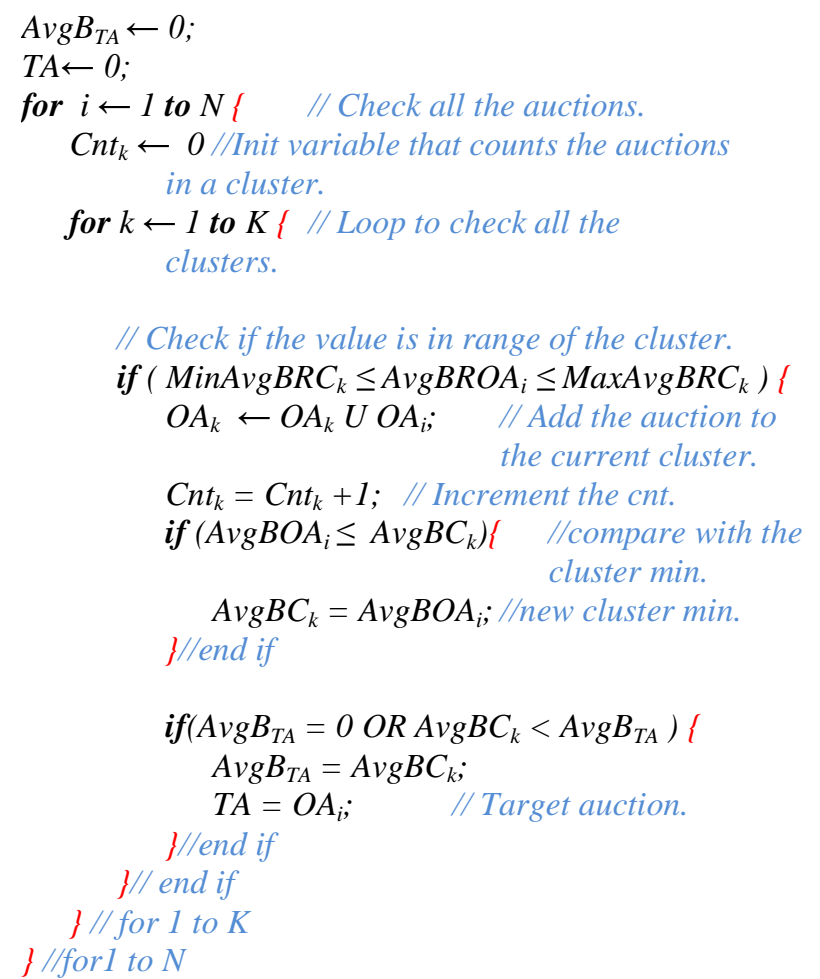

Fig. 2. Bid Mapper and Selector Algorithm (BMS)

3) Value Assessment: The value of the auctioned item in the target auction is assessed by predicting the closing price of the auction. The closing price prediction of the auction helps bidders in setting the true value of the item and in turn finalizing their maximum valuation of the auctioned item. The closing price prediction task is treated by using multiple regression technique [26] . 


\section{B. Phase 2:Final Price Prediction}

The second phase of the ADBA agent predicts the final price of the target auction by designing bidding strategies for the bidders. These bidding strategies are designed based on the output of the first phase for the bidders possessing different bidding behaviors. The bidding behaviors which depend on both the single or multiple bid placements and the time of bid placements are used to determine the various types of bidders (Fig. 3). The bidders who place a single bid are identified as Mystical and Sturdy bidders. The Mystical bidders place a single bid in the closing moments (last five seconds) of the auction and the Sturdy bidders place a single bid in the last five minutes of the auction. The bidders who place multiple bids in the last hour of the auction are identified as Strategic bidders and these bidders increase their bid amount strategically, based on the bids placed by the other participants.

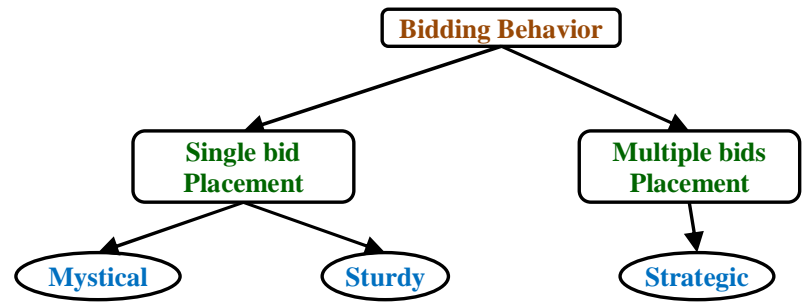

Fig. 3. Types of Bidders

These bidders commonly exhibit two types of behavior: firstly, they may be desperate to win the item, and secondly, they may be willing to bargain for that item. The bidders exhibiting these behaviors are designated as Ambitious and Sophisticated bidders respectively.

The bidding strategies are designed for the aforementioned bidding behaviors of buyers. The purpose of designing these bidding strategies is to determine how to compute bid amount at a particular moment of time. This bid is the maximum value that an agent is willing to pay at that particular moment in time. An agent negotiates this value based on bidding characteristics such as auction's attributes, bidder's own attitude and other bidder's attitude. Auctions' attributes has been considered while predicting the initial price of the auction during the first phase of the ADBA agent. Now we compute bid amount at a particular moment in time using bidder's own attitude and other bidder's attitude. We consider main two types of bidder's attitude towards achieving the goal; desperate to get the auctioned item (Ambitious bidders) or he has a desire of bargain to get the item (Sophisticated bidders). Ambitious bidder starts with higher bids to maximize its chances to win the auction, on the other hand, Sophisticated bidder starts with lower bids and slowly increments his bids towards its maximum willingness to pay near to the closing moments of the auction.

Other bidders' attitude is used to assess competition in the auction by exploiting their previous bids (competing bids) in the auction. Bidders update their bids at a particular moment in time based on the other's bids [27]. When the previous offers placed by other bidders (competing bids) are higher and rate of change of bids is higher, the bidder makes higher bids with more bid increments to win the auction indicating increased competition (Fig 4). In addition, remaining duration left till the auction closure is another important factor which affects the competition in the auction. The bidders make quick decisions towards end of the auction due to the time pressure. Time pressure increases arousals and the bidders bid beyond their limits as end point approaches, when there is little time left [28]. This augments the competition amongst auction participants. The competition increases as the remaining duration left of auction decreases (Fig. 5).

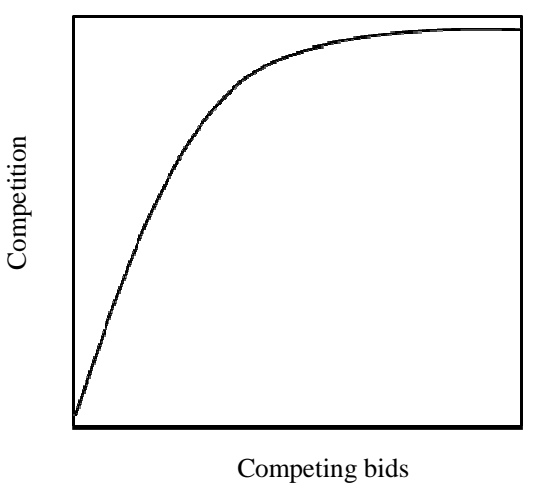

Fig. 4. Competition versus competing bids

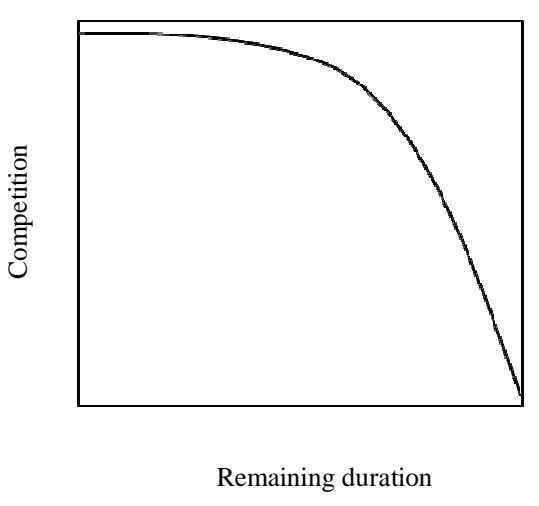

Fig. 5. Competition versus remaining duration

The bidding agent models the bid amount based on the initial price $\left(p_{i}\right)$ and using Mamdani's Method for fuzzy relations and compositional rule of inference for designing bidding strategies of buyers [25]. The bid increment for the auction is calculated based on the competition in an auction and the bidding attitude of different bidding behavior of buyers (where bid increment $(\Delta P)$ is the amount by which the bidder raises the current bid (initial bid $p_{i}$ )). We assess 
competition which depends on remaining duration of the auction and the previous offers made by other auction participants and then bid increment is calculated for different bidding behavior of bidders.

1) Competition assessment: Let $C$ is the competition having fuzzy set of values as $c_{1}, c_{2}, \ldots \ldots \ldots c_{n}, D$ is the remaining duration having fuzzy set of values as $d_{1}, d_{2}, \ldots \ldots . . d_{n}$ and $\mathrm{B}$ is the competing bids having fuzzy set of values $b_{1}, b_{2}, \ldots \ldots . . b_{n}$. According to Mamdani's Direct Method [19] we can find adaptability $n$ no. of rules $w_{l}$, $w_{2}, \ldots \ldots . w_{n}$ as follows

$$
\begin{aligned}
& w_{1}=\mu d_{1}(D) \quad V \mu b_{1}(B) \\
& w_{2}=\mu d_{2}(D) \quad \vee \mu b_{2}(B) \\
& \ldots \ldots \ldots \ldots \ldots \ldots \ldots \ldots \ldots \ldots \ldots \ldots \ldots \ldots \ldots \ldots \ldots \\
& w_{n}=\mu d_{n}(D) \quad \vee \mu b_{n}(B)
\end{aligned}
$$

Then the competition is assessed for each rule as follows

$$
\begin{aligned}
& \mu c^{\prime}{ }_{1}(C)=w_{1} V \mu c_{1} \\
& \mu c^{\prime}{ }_{2}(C)=w_{2} V \mu c_{2} \\
& \mu c^{\prime}{ }_{n}(C)=w_{n} V \mu c_{n}
\end{aligned}
$$

These rules are aggregated for the final competition evaluation

$$
\mu c(C)=\mu c_{1}^{\prime}(C) \wedge \mu c_{2}^{\prime}(C) \wedge \ldots \ldots \ldots \ldots \ldots \ldots \ldots \ldots \ldots c_{n}^{\prime}(C)
$$

Definite value of the competition is found by applying center of gravity of the fuzzy set refer to (2) as follows

$$
C=\int \mu c(C) C d C / \int \mu c(C) d C
$$

2) Bid determination: Now we calculate the bid increment $\Delta P$ for the auction based on the attitude and assessed competition by applying Mamdani's Method for fuzzy relations and compositional rule of inference. Let $\Delta P$ have the fuzzy set values as $p_{1}, p_{2}, \ldots \ldots \ldots p_{n}, E$ is the attitudes having fuzzy set of values as $e_{1}, e_{2} \ldots \ldots \ldots e_{n}$ and $C$ is the competition having fuzzy set of values as $c_{1}, c_{2}, \ldots \ldots \ldots c_{n}$.

\section{Here, premise 1: IF $c$ is $C$ and e is ETHEN $p$ is $\triangle P$ premise2: $\quad c$ is $C^{\prime}$ and e is $E^{\prime}$ consequence: $p$ is $\triangle P^{\prime}$}

where $C, C^{\prime}, E, E^{\prime}, \Delta P$ and $\Delta P^{\prime}$ are fuzzy sets. As per the mechanism of fuzzy reasoning, we infer " $p$ is $\Delta P$ " when the condition " $c$ is $C$ ' and $e$ is $E$ '" is given for the rule " $I F$ $c$ is $C$ and $e$ is E THEN $p$ is $\triangle P^{\prime \prime}$.

Using fuzzy relations approach, we first convert the $I F$ THEN rule in premise 1 into the fuzzy relation $R_{C}$ and $E \rightarrow \Delta P$. Then by applying compositional operation, we infer conclusion $\Delta P$ ' from the fuzzy relations $R_{C \text { and } E \rightarrow \Delta P}$ and the condition "c is $C$ ' and e is $E^{\prime \prime}$ of premise 2 (Fig. 6).

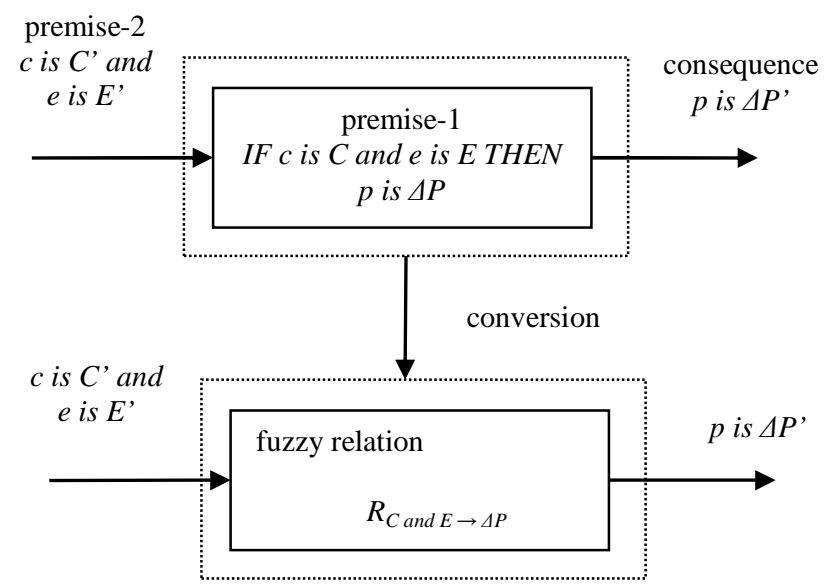

Fig. 6. Fuzzy reasoning by using fuzzy relations and the compositional rule of inference

According to Mamdani's Method for fuzzy relations and compositional rule of inference the rule $e_{i}$ and $c_{j} \rightarrow p_{k}$ is described by

$$
R=\int_{E \times C \times \Delta P} \frac{\left(\mu e_{i}(E) \wedge \mu c_{j}(C) \wedge \mu p_{k}(\Delta P)\right)}{(E, C, \Delta P)}
$$

The conversion refer to (4) is based on Cartesian product such as

$$
e_{i} \text { and }_{j} \rightarrow p_{k}=e_{i} \wedge c_{j} \wedge p_{k}
$$

The conversion by using membership value form is given as follows

$$
\mu_{R}(E, C, \Delta P)=\mu e_{i}(E) \wedge \mu c_{j}(C) \wedge \mu p_{k}(\Delta P)
$$

For $n$ number of rules, the compiled fuzzy relation $R$ is given as

$$
R=R_{1} \cup R_{2} \cup \ldots \ldots \ldots \cup R_{n}=\bigcup_{i=1}^{n} R_{i}
$$

For the input of fuzzy sets $E^{\prime}$ on $E$ and $C^{\prime}$ on $C$, the output fuzzy set $\Delta P^{\prime}$ on $\Delta P$ can be obtained as follows

$$
\Delta P^{\prime}=\left(E^{\prime} \text { and } C^{\prime}\right) \circ R=E^{\prime} \circ\left(C^{\prime} \circ R\right)=C^{\prime} \circ\left(E^{\prime} \circ R\right)
$$

Bid amount for the auction is calculated as

$$
\text { Bid_Amount }=p_{i}+\Delta P^{\prime}
$$

The fuzzy set $E^{\prime}$ depends on the selected bidding strategy for the bidding agent. The ambitious bidders have always higher attitude to win the auction than the sophisticated bidders because the ambitious bidders are desperate to get the item. Accordingly, the fuzzy set $E^{\prime}$ is described such that $E$ is high for ambitious bidders and low for sophisticated bidders. However, the sophisticated strategic bidders have 
higher attitude to win the auction as compared to the mystical and sturdy bidders of similar type, so $E$ is also high for sophisticated strategic bidders.

\section{EXPERIMENTS}

The performance of our bidding agent is assessed by undertaking an empirical evaluation of the automated dynamic bidding agent (ADBA) in two steps. First, the methodology for the initial estimation is validated, and second, the bidding strategies for different bidding behaviors of bidders are evaluated.

\section{A. Initial Price Estimation}

In the initial price estimation phase, an ongoing auction is selected for participation and its closing price is predicted. This estimated price acts as the initial price $\left(p_{i}\right)$ for designing the bidding strategies of bidders. The auction for participation is selected based on the bid mapping and selecting technique. The closing price of the selected auction is predicted by exploiting the clustering based approach [26]. In the proposed approach, the closing price of an online auction is predicted in two scenarios: first, by exploiting multiple linear regressions on whole input auction data, and second, by exploiting multiple linear regression on each cluster generated by applying the k-means algorithm on whole input auction data. The results are evaluated by comparing the root mean square errors (RMSEs) in both of these scenarios. Experimental results demonstrate fewer RMSEs for the prediction results when multiple linear regression is applied on each cluster rather than on whole input auction data [26].

\section{B. Evaluating the bidding strategies}

Final price of the target auction is predicted by designing bidding strategies for different bidding behavior of the bidders using fuzzy reasoning techniques. The bid increment $(\triangle P)$ for the bidders is calculated based on the bidding attitude of different bidding behavior of buyers and the competition in an auction. The performance of the bidding agent is compared with the classic model of the bidding agent in which the bidding attitude of bidders dominates over the competition in an auction while calculating the bid increment.

To compute $\Delta P$, the linguistic variables for the bidder's attitude and competition assessment are chosen as discussed in section II. The bidding strategies are analyzed by considering following set of rules for logic using various fuzzy sets.

Rule 1: IF attitude of agent to win the auction is $E_{l}$ AND competition in the market for that product is $C_{l}$ THEN the bid increment will be $P_{l}$

Rule 2: IF attitude of agent to win the auction is $E_{1}$ AND competition in the market for that product is $C_{2}$ THEN the bid increment will be $P_{2}$

Rule 3: IF attitude of agent to win the auction is $E_{2}$ AND competition in the market for that product is $C_{l}$ THEN the bid increment will be $P_{2}$
Rule 4: IF attitude of agent to win the auction is $E_{2}$ AND competition in the market for that product is $C_{2}$ THEN the bid increment will be $P_{3}$

These fuzzy sets represent the linguistic variables as follows: attitudes low as $E_{1}$ and high as $E_{2}$, competition low as $C_{1}$ and high as $C_{2} . P_{1}, P_{2}$ and $P_{3}$ are the bid increments based on the characteristics of the auction, where $P_{3} \geq P_{2} \geq P_{1}$. We assume that the set of attitudes for buying any item as $E=\left[e_{1}, e_{2}, e_{3}\right]=[0,0.5,1.0]$ and set of competition for the good in the market as $C=\left[c_{1}, c_{2}, c_{3}\right]=[0,0.5,1.0]$. The fuzzy sets used in the preceding four rules can be quantized as shown in Fig 7.

$$
\begin{array}{ll}
E_{1}=[1.0,0.5,0] C 1=[1.0,0.5,0] & P_{1}=[1.0,0,0] \\
E_{2}=[0,0.5,1.0] C 2=[0,0.5,1.0] & P_{2}=[0,1.0,0] \\
& P_{3}=[0,0,1.0]
\end{array}
$$
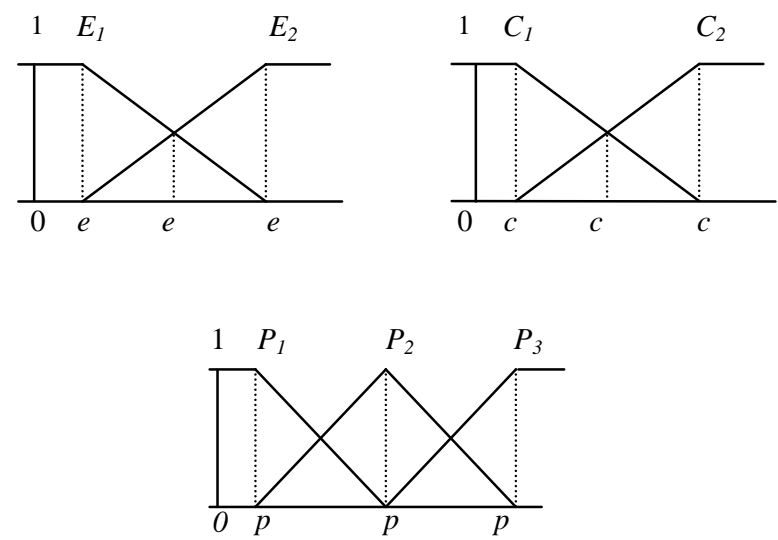

Fig. 7. Fuzzy Sets for the Bidding Logic

The competition for these bidding strategies is assessed based on the remaining duration left and the bids placed by other participants (competing bids). As the bidding strategies select bids based on its own attitude as well as on the bids placed by the other participants, these strategies will be successful when the bidder has a desire to bargain behavior. So in this set of experiments, we will discuss the performance of Sophisticated agents which act strategically based on the bids placed by the other participants.

For each rule given above, fuzzy relations $(R 1, R 2, R 3$ and $R 4$ ) are constructed using Mamdani's method for fuzzy relations and compositional rule of inference. R1 is shown as in Fig 8 and the total fuzzy relation $\mathrm{R}$ is given as in Fig 9.

The output fuzzy set $\Delta P^{\prime}$ on $\Delta P$ is calculated for different bidding strategies of bidders using input fuzzy sets $E^{\prime}$ on $E$ and $C^{\prime}$ on $C$ by applying Mamdani's compositional rule of Inference for different levels of competition (low and high). A definite value of the bid increment is calculated by defuzzifying $\Delta P^{\prime}$ using a center of gravity with the weighted mean method. 


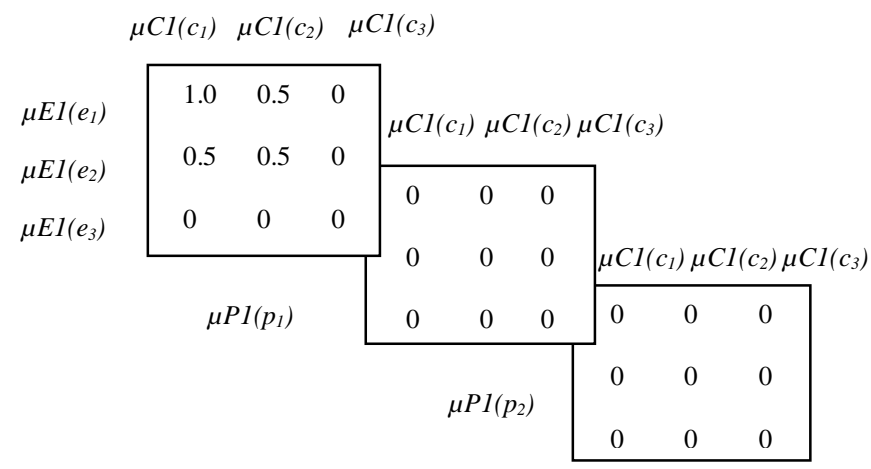

Fig. 8. Fuzzy relation for the fuzzy rule R1

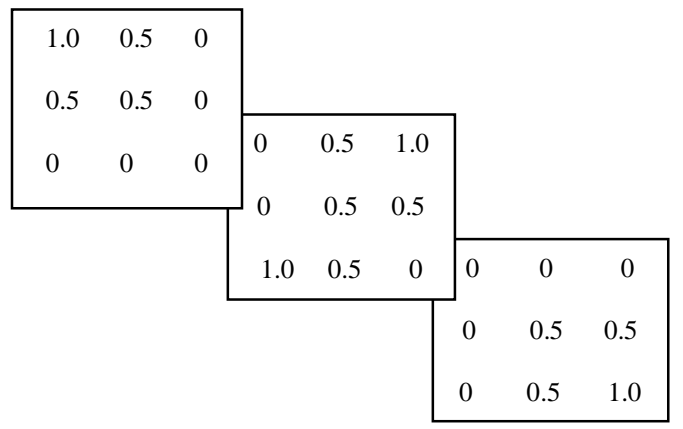

Fig. 9. Total fuzzy relation $\mathrm{R}$

The fuzzy bidding strategies are assessed in different bidding environments of varying bid rates of auctions. Let Bidder $r_{1}$ be the fuzzy bidder agent designed in section II and Bidder $_{2}$ be the classic model of the bidder agent. These bidders with varying bidding strategies compete against one another for each type of auction separately. The success rate percentage and the expected utility of the bidding agents are used as the performance measures.

Success rate percentage $=R_{\text {success }}=r_{\text {success }} * 100$

where $r_{\text {success }}$ be the success rate and $r_{\text {success }}=N_{\text {win }} / N_{\text {total }}$, $N_{\text {win }}$ be the number of auctions won by the agent and $N_{\text {total }}$ be the total number of auctions.

\section{Expected utility $=U_{\text {exp }}=U_{\text {win }} * r_{\text {success }}$}

where $U_{\text {win }}$ be the utility of the winning agents and $U_{\text {win }}=$ $\left(p_{r}-v_{i}\right) / p_{r}$, where $p_{r}$ be the reservation price (maximum willingness to pay) and $v_{i}$ be the winning price of the auction.
$R_{\text {success }}$ and $U_{\text {exp }}$ of Bidder Bid $_{1}$ and Bider 2 agents with different bidding behaviors are averaged over auctions with varying bid rates. As can be seen from the Table I, Bidder ${ }_{1}$ with mystical, sturdy and strategic behavior outperform the classic Bidder ${ }_{2}$ agents with mystical, sturdy and strategic behaviors respectively with respect to the $R_{\text {success }}$ and $U_{\text {exp }}$.

TABLE I.

SuCCESS RATE PERCENTAGE AND EXPECTED UTILITY COMPARISON OF BIDDER AGENTS

\begin{tabular}{|c|c|c|c|c|}
\hline \multirow{2}{*}{$\begin{array}{c}\text { Bidding } \\
\text { behavior }\end{array}$} & \multicolumn{2}{|c|}{ Success rate \%age } & \multicolumn{2}{c|}{ Expected utility } \\
\cline { 2 - 5 } & Bidder $_{1}$ & Bidder $_{2}$ & Bidder $_{1}$ & Bidder $_{2}$ \\
\hline Mystical & 41.67 & 8.33 & 0.2 & 0.002 \\
\hline Sturdy & 83.33 & 8.33 & 0.51 & 0 \\
\hline Strategic & 41.67 & 16.67 & 0.17 & 0.002 \\
\hline
\end{tabular}

\section{CONCLUSIONS}

In this paper we presented an automated dynamic bidding agent (ADBA) using fuzzy reasoning techniques to assist bidders in making decisions by designing bidding strategies as per their bidding behaviors. The bidding agent primarily performs three tasks; first, it decides which auction to bid in, secondly, assesses the value of the item in the selected auction, and finally, models the bid amount for a given bidding behavior of the bidders. Auction selection and the value assessment adopt a clustering based bid mapping and selection approach. The ADBA agent forecasts the final price of the selected ongoing auction by designing the bidding strategies based on the bidding behavior of the bidders. Bidding strategies are designed using Mamdani's Method for fuzzy relations and compositional rule of inference. The ADBA agent that preserves a balance between the bidding attitude of the bidders and the competition in the auction outperforms the classic model of final price prediction in terms of their success rate and the expected utility. Also, the improvement in the error measure for each cluster gives support in favor of the proposed clustering based bid mapping and selection approach of the value assessment of the item.

\section{REFERENCES}

[1] A. Ockenfels, D. H. Reiley, and A. Sadrieh, "Online auctions," in Economics and Information Systems Handbook, ed. Amsterdam: Elsevier Science, 2006, pp. 571-622.

[2] E. Haruvy, "Internet auctions," Foundations and Trends ${ }^{\circledR}$ in Marketing, vol. 4, pp. 1-75, 2009.

[3] Y. H. Park and E. T. Bradlow, "An integrated model for bidding behavior in Internet auctions: Whether, who, when, and how much," Journal of Marketing Research, vol. 42, pp. 470-482, 2005.

[4] W. Jank and S. Zhang, "An automated and data-driven bidding strategy for online auctions," Journal of Computing, vol. 23, pp. 238253, 2011.

[5] P. Anthony, W. Hall, V. Dang, and N. Jennings, "Autonomous agents for participating in multiple online auctions," 2001.

[6] A. Greenwald and P. Stone, "Autonomous bidding agents in the trading agent competition," IEEE Internet Computing, vol. 5, pp. 52$60,2001$.

[7] E. Haruvy and P. T. L. Popkowski Leszczyc, "Internet Auctions," Foundations and Trends ${ }^{\circledR}$ in Marketing, vol. 4, pp. 1-75, 2010.

[8] R. Bapna, P. Goes, A. Gupta, and Y. Jin, "User heterogeneity and its impact on electronic auction market design: An empirical exploration," Mis Quarterly, vol. 28, pp. 21-43, 2004. 
[9] H. Shah, N. Joshi, A. Sureka, and P. Wurman, "Mining eBay: Bidding strategies and shill detection," WEBKDD 2002-MiningWeb Data for Discovering Usage Patterns and Profiles, pp. 17-34, 2003.

[10] J. Trevathan and W. Read, "Detecting shill bidding in online english auctions," Handbook of Research on Social and Organizational Liabilities in Information Security, 2008.

[11] L. Du, Q. Chen, and N. Bian, "An Empirical Analysis of Bidding Behavior in Simultaneous Ascending-Bid Auctions," in International Conference on E-Business and E-Government (ICEE), 2010, pp. 249251.

[12] E. B. Rasmusen, "Strategic implications of uncertainty over one's own private value in auctions," The BE Journal of Theoretical Economics, vol. 6, p. 7, 2006.

[13] A. Ockenfels and A. E. Roth, "Late and multiple bidding in second price Internet auctions: Theory and evidence concerning different rules for ending an auction," Games and Economic Behavior, vol. 55, pp. 297-320, 2006.

[14] L. Xuefeng, L. Lu, W. Lihua, and Z. Zhao, "Predicting the final prices of online auction items," Expert Systems with Applications, vol. 31, pp. 542-550, 2006.

[15] V. Nikolaidou and P. Mitkas, "A Sequence Mining Method to Predict the Bidding Strategy of Trading Agents," in Agents and Data Mining Interaction, ed, 2009, pp. 139-151.

[16] D. D. Kehagias and P. A. Mitkas, "Efficient E-Commerce Agent Design Based on Clustering eBay Data," in International Conferences on Web Intelligence and Intelligent Agent Technology Workshops, 2007 IEEE/WIC/ACM 2007, pp. 495-498.

[17] D. Heijst, R. Potharst, and M. Wezel, "A support system for predicting ebay end prices," Econometric Institute Report, 2006.

[18] R. Ghani and H. Simmons, "Predicting the end-price of online auctions," in Proceedings of the International Workshop on Data Mining and Adaptive Modelling Methods for Economics and Management, Pisa, Italy, 2004.

[19] S. Zhang, W. Jank, and G. Shmueli, "Real-time forecasting of online auctions via functional k-nearest neighbors," International Journal of Forecasting, vol. 26, pp. 666-683, 2010.

[20] D. Van Heijst, R. Potharst, and M. Van Wezel, "A support system for predicting eBay end prices," Decision Support Systems, vol. 44, pp. 970-982, 2008.

[21] S. Wang, W. Jank, and G. Shmueli, "Explaining and forecasting online auction prices and their dynamics using functional data analysis," Journal of Business and Economic Statistics, vol. 26, pp. 144-160, 2008.

[22] D. Kehagias, A. Symeonidis, and P. Mitkas, "Designing pricing mechanisms for autonomous agents based on bid-forecasting," Electronic Markets, vol. 15, pp. 53-62, 2005.

[23] M. Dass, W. Jank, and G. Shmueli, "Maximizing bidder surplus in simultaneous online art auctions via dynamic forecasting," International Journal of Forecasting, vol. 27, pp. 1259-1270, 10// 2011.

[24] W. Jank and G. Shmueli, "Profiling price dynamics in online auctions using curve clustering," Smith School of Business, University of Maryland, Working paper2005.

[25] K. Tanaka and T. Niimura, An introduction to fuzzy logic for practical applications: Springer New York, 1997.

[26] P. Kaur, M. Goyal, and J. Lu, "Data mining driven agents for predicting online auction's end price," in IEEE Symposium on Computational Intelligence and Data Mining (CIDM), Paris, 2011, pp. 141-147.

[27] D. Ariely and I. Simonson, "Buying, bidding, playing, or competing? Value assessment and decision dynamics in online auctions," Journal of Consumer Psychology, vol. 13, pp. 113-123, 2003.

[28] G. Ku, D. Malhotra, and J. K. Murnighan, "Towards a competitive arousal model of decision-making: A study of auction fever in live and Internet auctions," Organizational Behavior and Human Decision Processes, vol. 96, pp. 89-103, 2005. 